\title{
Treatable traits in an English cohort: prevalence and predictors of future decline in lung function and quality of life in COPD
}

\author{
Muhammad Rehan Sarwar (10) ${ }^{1}$, Vanessa Marie McDonald (103, 2,4 , \\ Michael John Abramson (1) ${ }^{5}$, Eldho Paul $\mathbb{1}^{5}$ and Johnson George (1) 1
}

Affiliations: ${ }^{1}$ Centre for Medicine Use and Safety, Faculty of Pharmacy and Pharmaceutical Sciences, Monash University, Melbourne, Australia. ${ }^{2}$ National Health and Medical Research Council Centre for Research Excellence in Severe Asthma and Centre of Excellence in Treatable Traits, The University of Newcastle, Callaghan, Australia. ${ }^{3}$ The Priority Research Centre for Healthy Lungs, School of Nursing and Midwifery, Callaghan, Australia. ${ }^{4}$ Dept of Respiratory and Sleep Medicine, John Hunter Hospital, Hunter Medical Research Institute, Newcastle, Australia. ${ }^{5}$ School of Public Health and Preventive Medicine, Monash University, Melbourne, Australia.

Correspondence: Johnson George, Centre for Medicine Use and Safety, Faculty of Pharmacy and Pharmaceutical Sciences, Monash University, 381 Royal Parade, Parkville, VIC 3052, Australia. E-mail: johnson.georgedmonash.edu

\section{ABSTRACT}

Background: "Treatable traits (TTs)" is a precision medicine approach for facilitating multidimensional assessment of every patient with chronic airway disease, in order to determine the core traits associated with disease outcomes where targeted treatments may be applied.

Objectives: To determine the prevalence of TTs in chronic obstructive pulmonary disease (COPD) and which traits predict future decline in lung function and quality of life (QoL).

Methods: A 4-year longitudinal evaluation was conducted using data from 3726 participants in the English Longitudinal Study of Ageing (ELSA). TTs were identified based on published recommendations. Traits that predicted decline in lung function and QoL were analysed using generalised estimating equations.

Results: Overall, 21 TTs, including pulmonary $(n=5)$, extra-pulmonary $(n=13)$ and behavioural/lifestyle risk-factors $(n=3)$ were identified. In multivariate analyses, the traits of chronic bronchitis $(\beta-0.186,95 \%$ CI -0.290 to -0.082$)$, breathlessness $(\beta-0.093,95 \% \mathrm{CI}-0.164$ to -0.022$)$, underweight $(\beta-0.216,95 \% \mathrm{CI}$ -0.373 to -0.058$)$, sarcopenia $(\beta-0.162,95 \%$ CI -0.262 to -0.061$)$ and current smoking $(\beta-0.228,95 \%$ $\mathrm{CI}-0.304$ to -0.153$)$ predicted decline in forced expiratory volume in $1 \mathrm{~s}\left(\mathrm{FEV}_{1}\right)$. Of the seven traits that predicted decline in QoL, depression $(\beta-7.19,95 \% \mathrm{CI}-8.81$ to -5.57$)$ and poor family and social support $(\beta-5.12,95 \%$ CI -6.65 to -3.59$)$ were the strongest.

Conclusion: The core TTs of COPD associated with a decline in lung function and QoL were identified. Targeting these impactful traits with individualised treatment using a precision medicine approach may improve outcomes in people with COPD.

@ERSpublications

Targeting the traits of chronic bronchitis, breathlessness, underweight, sarcopenia, depression, smoking, and poor family and social support may significantly improve health outcomes in people with COPD https://bit.ly/3cLHIPV

ite this article as: Sarwar MR, McDonald VM, Abramson MJ, et al. Treatable traits in an English cohort: prevalence and predictors of future decline in lung function and quality of life in COPD. ERJ Open Res 2021; 7: 00934-2020 [https://doi.org/10.1183/23120541.00934-2020].

This article has supplementary material available from openres.ersjournals.com.

Received: 14 Dec 2020 | Accepted after revision: 29 Jan 2021

Copyright $\odot$ The authors 2021. This version is distributed under the terms of the Creative Commons Attribution NonCommercial Licence 4.0. For commercial reproduction rights and permissions contact permissions@ersnet.org 


\section{Introduction}

Chronic obstructive pulmonary disease (COPD) is a heterogeneous disease complicated by comorbidities and exacerbations, making its management complex. Despite the use of many evidence-based approaches to management, the burden from COPD exacerbations and symptoms continues [1]. This has led to calls for new approaches to COPD management [2-4]. "Treatable traits" (TTs), a precision medicine strategy for chronic airway diseases first proposed by AGUsti et al. [3], is one such approach. The TTs strategy encompasses the multidimensional assessment of every patient (including a review of clinical history and a physical examination, as well as spirometry, a determination of risk factors, and exhaled nitric oxide fraction $\left(F_{\mathrm{ENO}}\right)$ and blood eosinophil levels) for identification of characteristics relating to genes, clinical and inflammatory phenotypes, and psychosocial factors in that individual. A personalised management approach can then be applied according to the traits identified [2]. Traits are classified under three domains: pulmonary, extra-pulmonary and behavioural/lifestyle risk factors [3].

The strategy of TTs supports a targeted approach that is widely applicable regardless of the specific airway disease diagnosis, in contrast to the "one size fits all" approach to management of airway diseases. Precision medicine could be a pivotal strategy for improved outcomes for patients with chronic airway diseases [3].

Previous studies in patients with chronic airway diseases identified that TTs such as anxiety and depression [5, 6], dysfunctional breathing [6], frequent chest infections [6], poor inhaler technique [6], smoking [7] and systemic inflammation [6,8] were associated with a greater decline in forced expiratory volume in $1 \mathrm{~s}\left(\mathrm{FEV}_{1}\right)$ and/or quality of life (QoL). However, which TTs matter most for optimising the management of COPD is still unclear. Moreover, there are scarce data from longitudinal studies investigating the prevalence of TTs among patients with COPD and their associations with lung function and QoL. To address this knowledge gap, the current analysis aimed to determine the prevalence of TTs among participants in a population-based cohort study, the English Longitudinal Study of Ageing (ELSA) and to determine which traits predict longitudinal decline in lung function and QoL.

\section{Methods}

Study design, setting and participants

ELSA is an ongoing cohort study evaluating the health and circumstances of people aged 50 years and over, born before 1952 and living in England [9]. The participants were recruited through multistage stratified probability sampling. Data were collected through interviews and self-completed questionnaires by a team of trained researchers who followed strict protocols. For this analysis we accessed data from ELSA, including 4-year follow-up data from Wave 2 (2004-2005) and Wave 4 (2008-2009). A total of 7666 respondents were interviewed by a nurse in Wave 2 and 8643 in Wave 4. Of these, participants with an asthma diagnosis (Wave $2(n=703)$; Wave $4(n=592))$ and those who had no valid respiratory function measurements (Wave $2(n=775)$; Wave $4(n=1019))$ were excluded. Of the remaining respondents (Wave 2 $(n=6188)$; Wave $4(n=7032)$ ), a final sample of 3726 people who were participants in both waves was included and used for all further analyses (figure 1).

\section{Data collection/measurements}

Socio-demographic characteristics

The following variables were recorded: gender, age (years), marital status (single/separated/widowed, living in a relationship), ethnicity (white, non-white) and socio-economic class based on employment (managerial and professional, intermediate, small employer, lower supervisory/technical and semi-routine occupations), according to the National Statistics Socio-economic Classification.

\section{Self-reported general health}

Participants self-rated their health status as "poor", "fair", "good", "very good" or "excellent" on a five-point Likert-type scale.

\section{Lung function/spirometry}

On Wave 2 and Wave 4, a trained nurse visited participants and performed (pre-bronchodilator) spirometry according to standardised criteria [10], using a portable Escort spirometer (Vitalograph Micro, Maids Moreton, UK) on both occasions, which automatically accounted for ambient temperature. A 1-L syringe was used for daily spirometer calibration [10]. This study included only those participants who were tested for lung function at baseline in 2004/2005 (Wave 2) and were followed-up in 2008/2009 (Wave 4). The Global Lung Function Initiative (GLI) 2012 spirometry task force reference equations [11] were used to derive the values for $\%$ predicted $\mathrm{FEV}_{1}$ and \% predicted forced vital capacity (FVC).

For the purposes of this analysis, individuals who had a fixed ratio of $\mathrm{FEV}_{1} / \mathrm{FVC}<0.70$ (spirometry data were not post-bronchodilator) and relevant symptoms (phlegm, dyspnoea and wheezing), but did not 


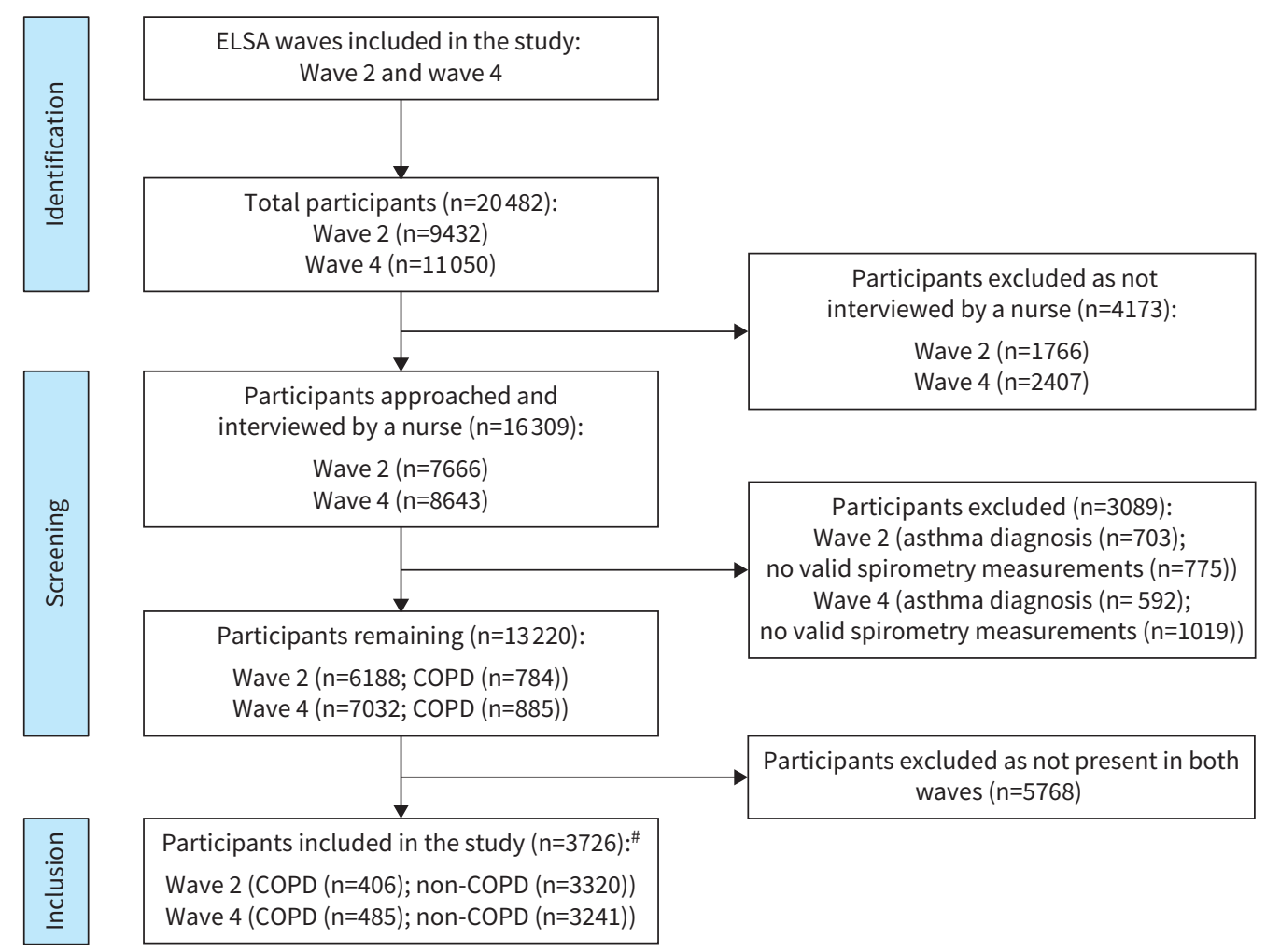

FIGURE 1 Schematic diagram explaining the flow of study participants. ELSA: English Longitudinal Study of Ageing; COPD: chronic obstructive pulmonary disease. \#: of the remaining 7452 participants, 3726 were common in both waves yielding a final sample of 3726 participants with 4-year follow-up.

self-report a diagnosis of asthma, were regarded as having COPD [12]. The severity of COPD was determined according to the Global Initiative for Chronic Obstructive Lung Disease (GOLD) classification system [13] and was categorised into "healthy/non-COPD" ( $\left.\mathrm{FEV}_{1} / \mathrm{FVC} \geqslant 0.7\right)$, "mild" ( $\mathrm{FEV}_{1} / \mathrm{FVC}<0.7$ and $\mathrm{FEV}_{1} \geqslant 80 \%$ pred), "moderate" ( $\mathrm{FEV}_{1} / \mathrm{FVC}<0.7$ and $50 \% \leqslant \mathrm{FEV}_{1}<80 \%$ pred), "severe" ( $\mathrm{FEV}_{1} / \mathrm{FVC}<0.7$ and $30 \% \leqslant \mathrm{FEV}_{1}<50 \%$ pred) or "very severe" ( $\mathrm{FEV}_{1} / \mathrm{FVC}<0.7$ and $\mathrm{FEV}_{1}<30 \%$ pred).

\section{Quality of life}

General QoL was assessed using the four-dimensional, 19-item control, autonomy, self-realisation and pleasure (CASP-19) scale [14]. Each item was measured on a four-point Likert-type scale (where: never=0, not often $=1$, sometimes $=2$ and often $=3$ ). The total CASP-19 score lies between 0 and 57, with higher scores suggesting greater well-being. CASP-19 is a validated tool explicitly developed for people in early old age and has been widely used in ageing surveys [14].

\section{Treatable traits in ELSA}

Potential TTs and corresponding diagnostic criteria were identified through a literature search $[1-4,15]$. Data for a total of 21 TTs (pulmonary $(n=5)$; extra-pulmonary $(n=13)$; behavioural/lifestyle $(n=3)$ ) could be characterised using the ELSA data set (table 1). Further details pertaining to the measurement of included TTs from the ELSA data set are presented in the supplementary material.

\section{Statistical analyses}

SPSS Statistics, version 23.0 (IBM Corp., Armonk, NY, USA) was used for data analysis. The distributions of continuous variables were assessed using Kolmogorov-Smirnov and Shapiro-Wilk tests. Baseline socio-demographic and health-related characteristics were summarised using frequencies, percentages and medians with interquartile ranges (IQRs). Characteristics, including TTs, were compared between COPD and non-COPD groups using Pearson's Chi-Squared test or the Mann-Whitney U-test, as appropriate.

Of all cases, $62.9 \%$ had no missing values for any variable; however, $37.1 \%$ of cases had missing value(s) for one or more variables. Little's missing completely at random (MCAR) test was used to establish the nature of missing data. Missing values were imputed using a Markov chain Monte Carlo multiple imputation method based on socio-demographic and clinical characteristics. Five data sets were imputed. 


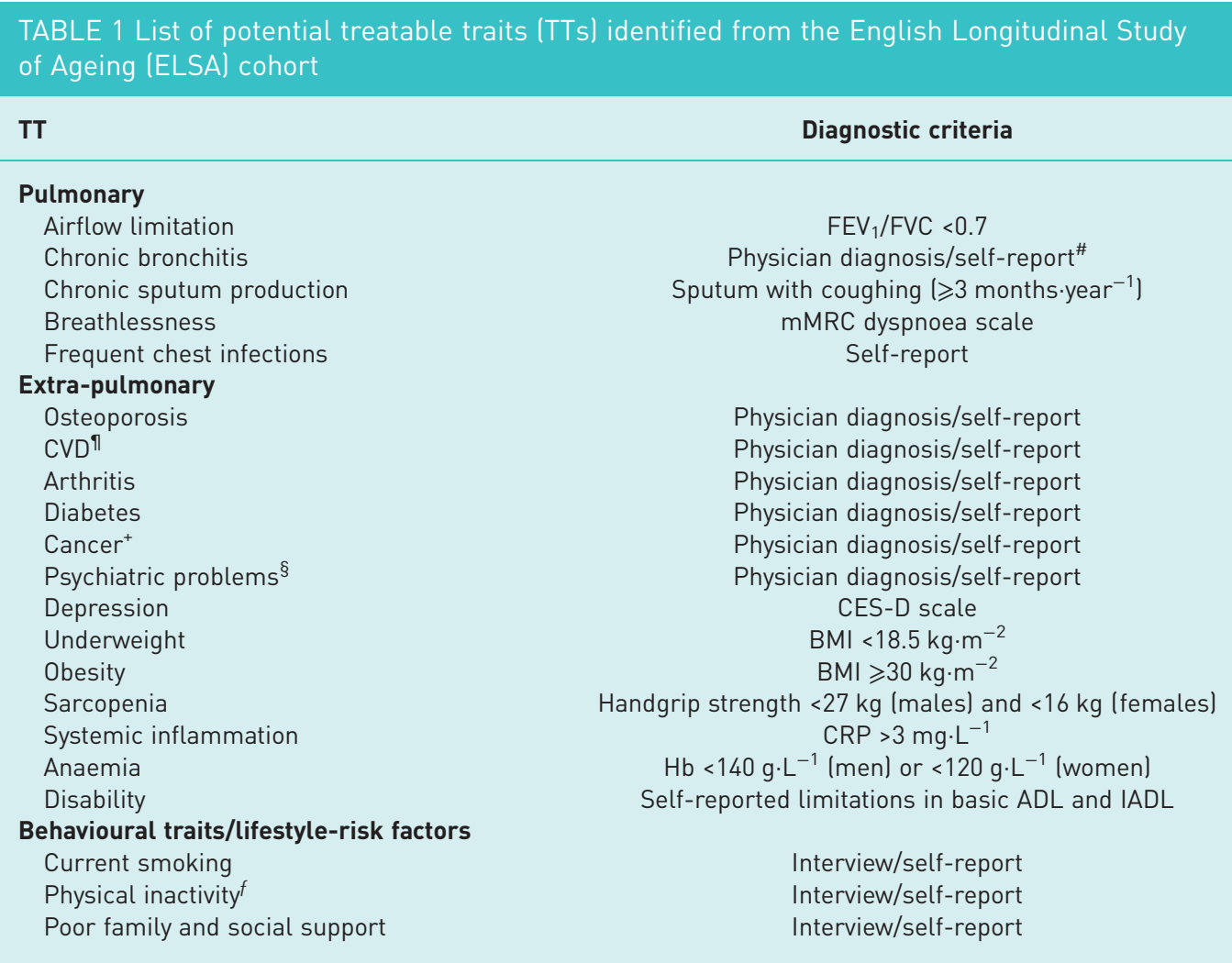

$\mathrm{FEV}_{1}$ : forced expiratory volume in $1 \mathrm{~s}$; FVC: forced vital capacity; mMRC: modified Medical Research Council; CVD: cardiovascular disease; CES-D: Centre for Epidemiological Studies-Depression; BMI: body mass index; CRP: C-reactive protein; $\mathrm{Hb}$ : haemoglobin; ADL: activities of daily living; IADL: instrumental activities of daily living. " : self-reported by the participants; П: including high blood pressure (BP), angina, myocardial infarction, heart attack, high cholesterol, congestive heart failure, stroke and heart murmur or abnormal heart rhythm; ${ }^{+}$: including cancers of the lung and breast, as well as colorectal cancers, lymphoma, leukaemia and melanoma; §: including hallucinations, anxiety, emotional problems, schizophrenia, psychosis, mood swings and bipolar disorder; ${ }^{f}$ : no mild, moderate or vigorous activity per week.

Each imputed data set was analysed, providing five sets of parameter estimates. Parameter estimates from each replication of analysis were averaged to provide a single estimate.

To assess the TTs that predicted longitudinal changes in lung function ( $\mathrm{FEV}_{1}$, both in $\mathrm{L}$ and \%pred) and QoL in the COPD and non-COPD groups, generalised estimating equations (GEEs) were used while controlling for potential confounders. We used two models for adjusting the potential confounders. The first (Model 1) included socio-demographic variables and TTs that had $\mathrm{p}<0.1$ from univariate analyses and the second (Model 2) included socio-demographic variables and TTs that had $\mathrm{p}<0.1$ from the multivariate analyses. "Quasi likelihood under independence" or "corrected quasi likelihood under independence" model criteria were compared between models to determine the best-working correlation structure. The GEE results were based on an independent correlation matrix for unadjusted analyses and upon an unstructured correlation matrix for the adjusted analyses (Model 1 and Model 2). All regression coefficients $(\beta)$ were reported along with $95 \%$ confidence intervals (CIs) and p-values. A two-sided p-value of less than 0.05 was considered statistically significant.

Complete case analyses were conducted within the GEE and presented as the primary analyses. Sensitivity analysis was performed using data obtained after multiple imputations to ensure robustness of the primary analysis. Furthermore, an additional sensitivity analysis was conducted for the non-COPD/control participants after excluding patients with airflow limitation and chronic bronchitis.

\section{Results}

Of the 3726 participants at baseline (Wave 2), 10.9\% had COPD (median age 67 years). Compared to those without COPD, participants with COPD were older, more likely to be single/separated/widowed, 
semi-routine employees and current smokers, as well as having fair or poor self-reported health status, limited physical activity, more severe dyspnoea and worse lung function (table 2).

\section{Prevalence of treatable traits}

All TTs had a higher prevalence among COPD participants in comparison to non-COPD participants (table 3). The following pulmonary TTs were significantly more prevalent among people with COPD: airflow limitation, breathlessness, chronic bronchitis, chronic sputum production and frequent chest infections. However, the COPD cohort also had a significantly higher prevalence of non-pulmonary traits including osteoporosis, arthritis, depression, sarcopaenia, systemic inflammation, disability, current smoking status, physical inactivity, and poor family and social support (table 3).

\section{Treatable traits and lung function}

The association between individual TTs and $\mathrm{FEV}_{1}$ is shown in table 4. Among individuals with COPD, univariate analyses showed that eight traits (chronic bronchitis, breathlessness, osteoporosis, depression,

\section{TABLE 2 Baseline socio-demographic and clinical characteristics of the cohort}

\begin{tabular}{|c|c|c|c|}
\hline Variable & COPD $(n=406)$ & Non-COPD $(n=3320)$ & p-value \\
\hline Male gender & $186(45.8)$ & $1526(46.0)$ & 0.954 \\
\hline Age years & 67.0 (59-73) & $63.0(57-70)$ & $<0.001$ \\
\hline Marital status & & & $<0.001$ \\
\hline Single/separated/widowed & $153(37.7)$ & 938 (28.3) & \\
\hline Living in a relationship & 253 (62.3) & $2382(71.7)$ & \\
\hline \multicolumn{4}{|l|}{ Ethnicity } \\
\hline White & 395 (97.3) & $3272(98.6)$ & 0.054 \\
\hline Socio-economic class & & & $<0.001$ \\
\hline Managerial and professional & $108(26.6)$ & $1209(36.4)$ & \\
\hline Intermediate & $53(13.1)$ & $467(14.1)$ & \\
\hline Small employers and own-account workers & $44(10.8)$ & $388(11.7)$ & \\
\hline Lower supervisory and technical & 50 (12.3) & $329(9.9)$ & \\
\hline Semi-routine occupations & $144(35.5)$ & $899(27.1)$ & \\
\hline Other & $7(1.7)$ & $28(0.8)$ & \\
\hline Self-reported health & & & $<0.001$ \\
\hline Excellent & $36(8.9)$ & $577(17.4)$ & \\
\hline Very good & 99 (24.4) & 1140 (34.3) & \\
\hline Good & $144(35.5)$ & $1071(32.3)$ & \\
\hline Fair & 93 (22.9) & $438(13.2)$ & \\
\hline Poor & $34(8.4)$ & $94(2.8)$ & \\
\hline Physical activity & & & $<0.001$ \\
\hline None & 25 (6.2) & $118(3.6)$ & \\
\hline Mild & 64 (15.8) & $373(11.2)$ & \\
\hline Moderate & $236(58.1)$ & $1684(50.7)$ & \\
\hline Vigorous & $81(20.0)$ & 1145 (34.5) & \\
\hline Dyspnoea & & & $<0.001$ \\
\hline Grade 0 & $172(42.4)$ & 2519 (75.9) & \\
\hline Grade 1 & $151(37.2)$ & $549(16.5)$ & \\
\hline Grade 2 & $34[8.4]$ & $90(2.7)$ & \\
\hline Grade 3 & $49(12.1)$ & $162(4.9)$ & \\
\hline Smoking status & & & $<0.001$ \\
\hline Never smoker & $100(24.6)$ & $1346(40.5)$ & \\
\hline Former smoker & $160(39.4)$ & $1518(45.7)$ & \\
\hline Current smoker & $146(36.0)$ & $456(13.7)$ & \\
\hline \multicolumn{4}{|l|}{ Spirometry measurements } \\
\hline $\mathrm{FEV}_{1} \mathrm{~L}$ & $1.7(1.1-2.3)$ & $2.5(2.0-3.1)$ & $<0.001$ \\
\hline FVC L & $3.1(2.3-3.8)$ & $3.2(2.6-4.0)$ & 0.001 \\
\hline PEF L. $\min ^{-1}$ & 286.5 (202.8-378.8) & 385 (307.0-493.0) & $<0.001$ \\
\hline $\mathrm{FEV}_{1} \%$ pred & $70.2(49.5-84.6)$ & $94.0(83.5-104.8)$ & $<0.001$ \\
\hline FVC \% pred & 92.2 (78.0-107.2) & $94.4(83.6-106.0)$ & 0.056 \\
\hline
\end{tabular}


TABLE 3 Prevalence of various treatable traits (TTS) at baseline by Global Initiative for Chronic Obstructive Lung Disease (GOLD) severity status

\begin{tabular}{|c|c|c|c|c|c|c|c|}
\hline TT & \multicolumn{5}{|c|}{ COPD } & $\begin{array}{l}\text { Non-COPD } \\
(n=3320)\end{array}$ & p-value \\
\hline \multicolumn{8}{|l|}{ Pulmonary } \\
\hline Airflow limitation & $129(100)$ & $172(100)$ & $74(100)$ & 31 (100) & $406(100)$ & $462(13.9)$ & $<0.001$ \\
\hline Breathlessness & 61 (47.3) & 102 (59.3) & 50 (67.6) & $16(51.6)$ & $229(56.4)$ & 789 (23.8) & $<0.001$ \\
\hline Frequent chest infections & $10(7.8)$ & $24(14.0)$ & 10 (13.5) & $2(6.5)$ & 46 (11.3) & $237(7.1)$ & 0.003 \\
\hline \multicolumn{8}{|l|}{ Extra-pulmonary } \\
\hline Osteoporosis & $6(4.7)$ & $13(7.6)$ & $9(12.2)$ & $2(6.5)$ & $30(7.4)$ & $149(4.5)$ & 0.010 \\
\hline CVD & 23 (17.8) & 43 (25) & 18 (24.3) & $8(25.8)$ & $92(22.7)$ & 742 (22.3) & 0.747 \\
\hline Arthritis & 52 (40.3) & 76 (44.2) & $30(40.5)$ & 10 (32.3) & $168(41.4)$ & $1130(34)$ & 0.003 \\
\hline Diabetes & 5 (3.9) & $14(8.1)$ & $6(8.1)$ & 1 (3.2) & $26(6.4)$ & $211(6.4)$ & 0.970 \\
\hline Obesity & 36 (27.9) & $45(26.2)$ & $26(35.1)$ & $8(25.8)$ & 115 (28.3) & $912(27.5)$ & 0.765 \\
\hline Sarcopenia & $6(4.7)$ & $14(8.1)$ & $6(8.1)$ & $2(6.5)$ & $29(7.1)$ & $157(4.7)$ & 0.035 \\
\hline Systemic inflammation & 44 (34.1) & 79 (45.9) & 35 (47.3) & 11 (35.5) & $168(41.4)$ & $985(29.7)$ & $<0.001$ \\
\hline Anaemia & $8(6.2)$ & $16(9.3)$ & 5 (6.8) & $2(6.5)$ & $31(7.6)$ & $223(6.7)$ & 0.473 \\
\hline Disability & 77 (59.7) & $131(76.2)$ & $58(78.4)$ & $22(71.0)$ & $288(70.9)$ & $1674(50.4)$ & $<0.001$ \\
\hline \multicolumn{8}{|l|}{ Behavioural/lifestyle } \\
\hline Current smoking & $33(25.6)$ & $73(42.4)$ & 27 (36.5) & $13(41.9)$ & $146(36.0)$ & $458(13.8)$ & $<0.001$ \\
\hline Physical inactivity & $3(2.3)$ & $12(7.0)$ & 10 (13.5) & $0(0.0)$ & $25(6.2)$ & $118(3.6)$ & 0.010 \\
\hline $\begin{array}{l}\text { Poor family and social } \\
\text { support }\end{array}$ & $27(20.9)$ & 37 (21.5) & 14 (18.9) & $8(25.8)$ & $86(21.2)$ & 574 (17.3) & 0.039 \\
\hline
\end{tabular}

Data are presented as $\mathrm{n}(\%)$. $\mathrm{p}$-Values in bold are statistically significant. COPD: chronic obstructive pulmonary disease; CVD: cardiovascular disease. ${ }^{\#}$ : COPD (overall) versus non-COPD.

underweight, sarcopaenia, disability and physical inactivity) were associated with a statistically and clinically significant decline in $\mathrm{FEV}_{1}$ (range: $-149 \mathrm{~mL}$ to $-682 \mathrm{~mL}$ ). In multivariate analyses, chronic bronchitis, breathlessness, underweight, sarcopaenia and current smoking were associated with a statistically and clinically significant decline in $\mathrm{FEV}_{1}$ (range: $-93 \mathrm{~mL}$ to $-228 \mathrm{~mL}$ ) (table 4). Results from the sensitivity analyses were quite similar (supplementary table S1A). The associations between individual TTs and \% predicted $\mathrm{FEV}_{1}$ are shown in supplementary tables S2A, S2B and S2C.

Among individuals without COPD, univariate analyses showed that 15 traits (airflow limitation, chronic bronchitis, breathlessness, osteoporosis, cardiovascular disease (CVD), arthritis, diabetes, cancer, depression, obesity, sarcopaenia, systemic inflammation, disability, current smoking and physical inactivity) were associated with a statistically and clinically significant decline in $\mathrm{FEV}_{1}$ (range: $-111 \mathrm{~mL}$ to $-555 \mathrm{~mL}$ ). In multivariate analyses, eight traits (breathlessness, diabetes, obesity, sarcopaenia, systemic inflammation, disability, current smoking and physical inactivity) were significantly associated with decline in $\mathrm{FEV}_{1}$ (range: $-36 \mathrm{~mL}$ to $-202 \mathrm{~mL}$ ) (table 4). Results from the sensitivity analyses were similar, except for the traits of chronic bronchitis and frequent chest infections that showed an association with a decline in $\mathrm{FEV}_{1}$ in the multivariate analyses (supplementary table S1A). Additional sensitivity analyses executed for non-COPD/control participants showed similar results (table 4), except for the trait of disability that did not show an association with a decline in $\mathrm{FEV}_{1}$ in the multivariate analyses (supplementary table $\mathrm{S} 1 \mathrm{~B}$ ).

\section{Treatable traits and quality of life}

The association between individual TTs and QoL decline is shown in table 5. Among individuals with COPD, univariate analyses showed that 13 traits (chronic bronchitis, chronic sputum production, breathlessness, CVD, arthritis, diabetes, depression, sarcopaenia, anaemia, disability, current smoking, 
TABLE 4 Treatable traits (TTS) associated with decline in forced expiratory volume in $1 \mathrm{~s}$ (FEV 1

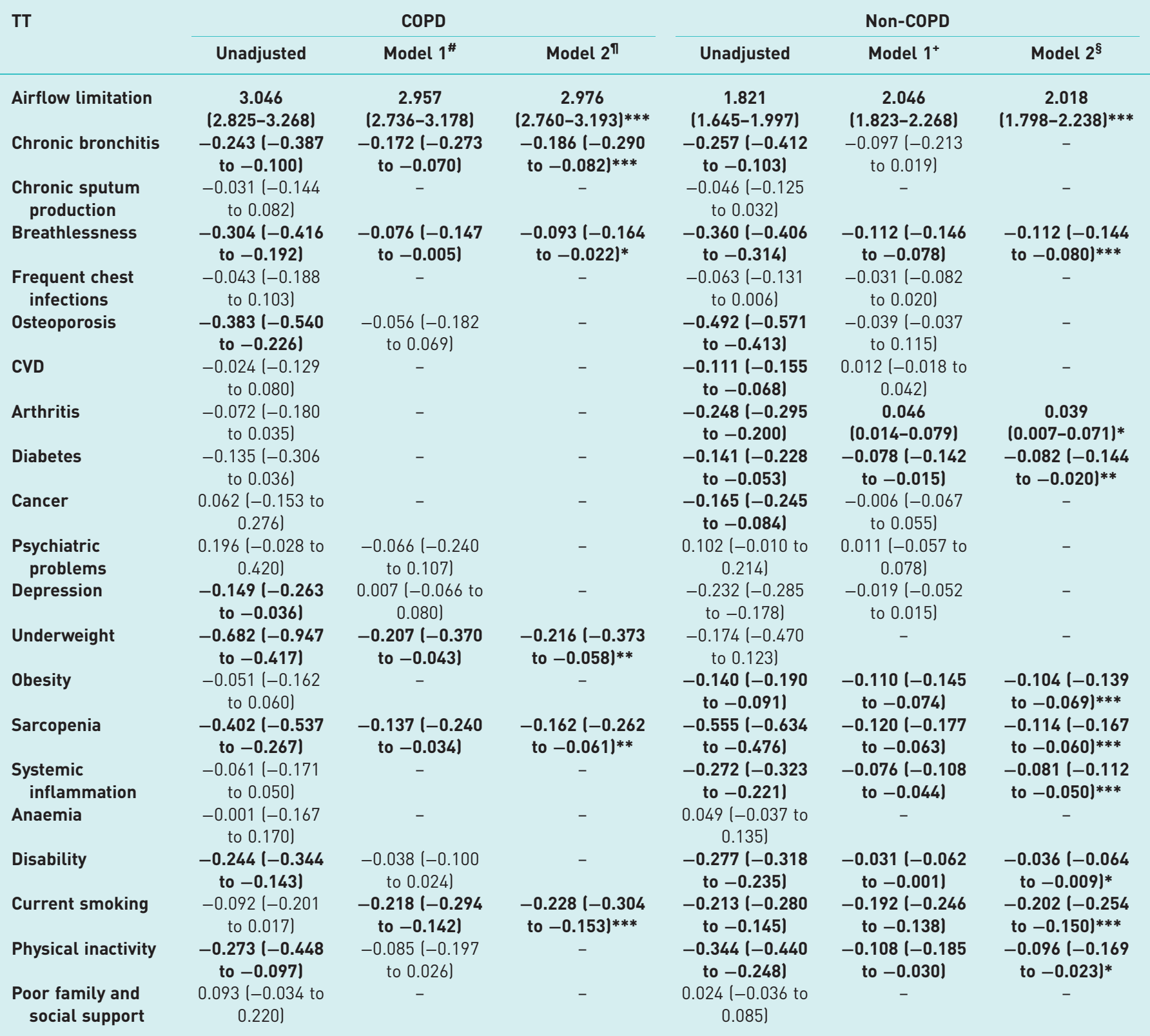

Data are presented as $\beta(95 \%$ Cl). Values in bold are statistically significant. COPD: chronic obstructive pulmonary disease; CVD: cardiovascular disease; $\mathrm{Cl}$ : confidence interval. \#: adjusted for sex, age, marital status, socioeconomic class and TTs ( $p<0.1)$ from the univariate analyses; " : adjusted for sex, age and TTs ( $p<0.1)$ from the multivariate analyses; ${ }^{+}$: adjusted for sex, age, marital status, ethnicity, socioeconomic class and TTs $(p<0.1)$ from the univariate analyses; ${ }^{\S}$ : adjusted for sex, age, marital status, ethnicity, socioeconomic class and TTs $(p<0.1)$ from the multivariate analyses. ${ }^{*}: p<0.05 ;{ }^{* *}: p<0.01 ;{ }^{* * *}: p<0.001$.

physical inactivity, and poor family and social support) were significantly associated with a decline in QoL score (range: -1.44 to -7.88 ). In the multivariate model, seven traits (chronic bronchitis, CVD, arthritis, depression, anaemia, disability, and poor family and social support) were significantly associated with a decline in QoL score (range: -1.52 to -7.19) (figure 2). Among these, the traits of depression and poor family and social support had the strongest associations with a decline in QoL (table 5). Results from the sensitivity analyses were similar except that chronic bronchitis and anaemia were not associated with a decline in QoL in the multivariate analyses. Nevertheless, chronic sputum production, breathlessness and current smoking showed associations with a decline in QoL in multivariate analyses (supplementary table S3A). 
TABLE 5 Treatable traits (TTS) associated with decline in quality of life (QoL)

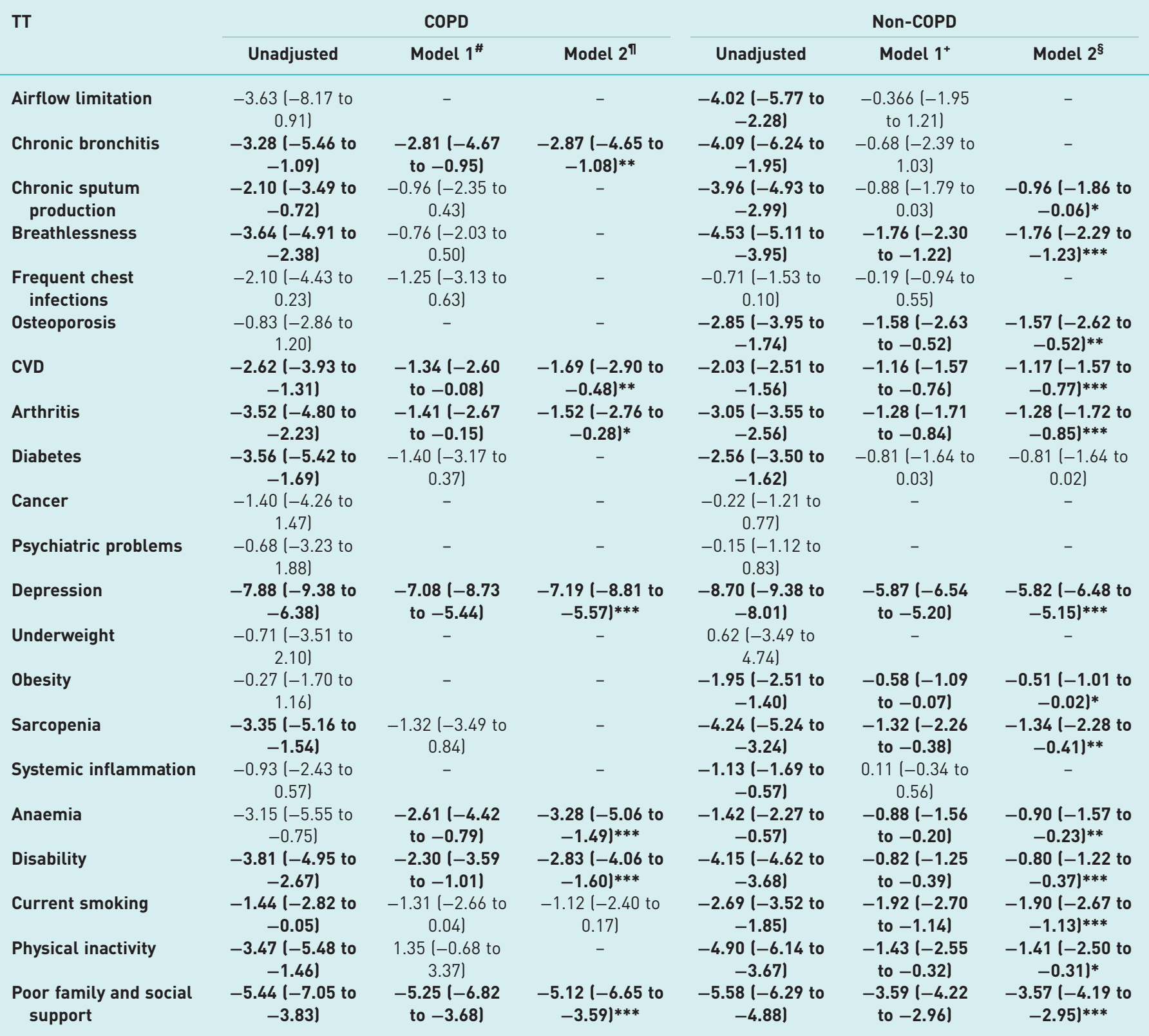

Data are presented as $\beta(95 \% \mathrm{CI})$. Values in bold are statistically significant. COPD: chronic obstructive pulmonary disease; CVD: cardiovascular disease; $\mathrm{Cl}$ : confidence interval. \#: adjusted for age, marital status, socioeconomic class and TTs ( $p<0.1)$ from the univariate analyses; ": adjusted for socioeconomic class and TTs $(p<0.1)$ from the multivariate analyses; ${ }^{+}$: adjusted for age, marital status, socioeconomic class and TTs $(p<0.1)$ from the univariate analyses; ${ }^{\S}$ : adjusted for age, socioeconomic class and TTs ( $\left.<<0.1\right)$ from the multivariate analyses. ${ }^{*}: p<0.05{ }^{* *}: p<0.01 ;{ }^{* * *}: p<0.001$.

Among individuals without COPD, 13 traits (chronic sputum production, breathlessness, osteoporosis, CVD, arthritis, depression, obesity, sarcopaenia, anaemia, disability, current smoking, physical inactivity, and poor family and social support) were significantly associated with a decline in QoL in multivariate analyses (range: -0.51 to -5.82 ) (table 5). Results from the sensitivity analyses were similar, except for anaemia that was not associated with a decline in QoL in multivariate analyses. Nevertheless, diabetes showed associations with a decline in QoL in multivariate analyses (supplementary table S3A). Additional sensitivity analyses executed for non-COPD/control subjects showed quite similar results (table 5), except for the traits of obesity and sarcopaenia that did not show an association with a decline in QoL in the multivariate analyses (supplementary table S3B). 


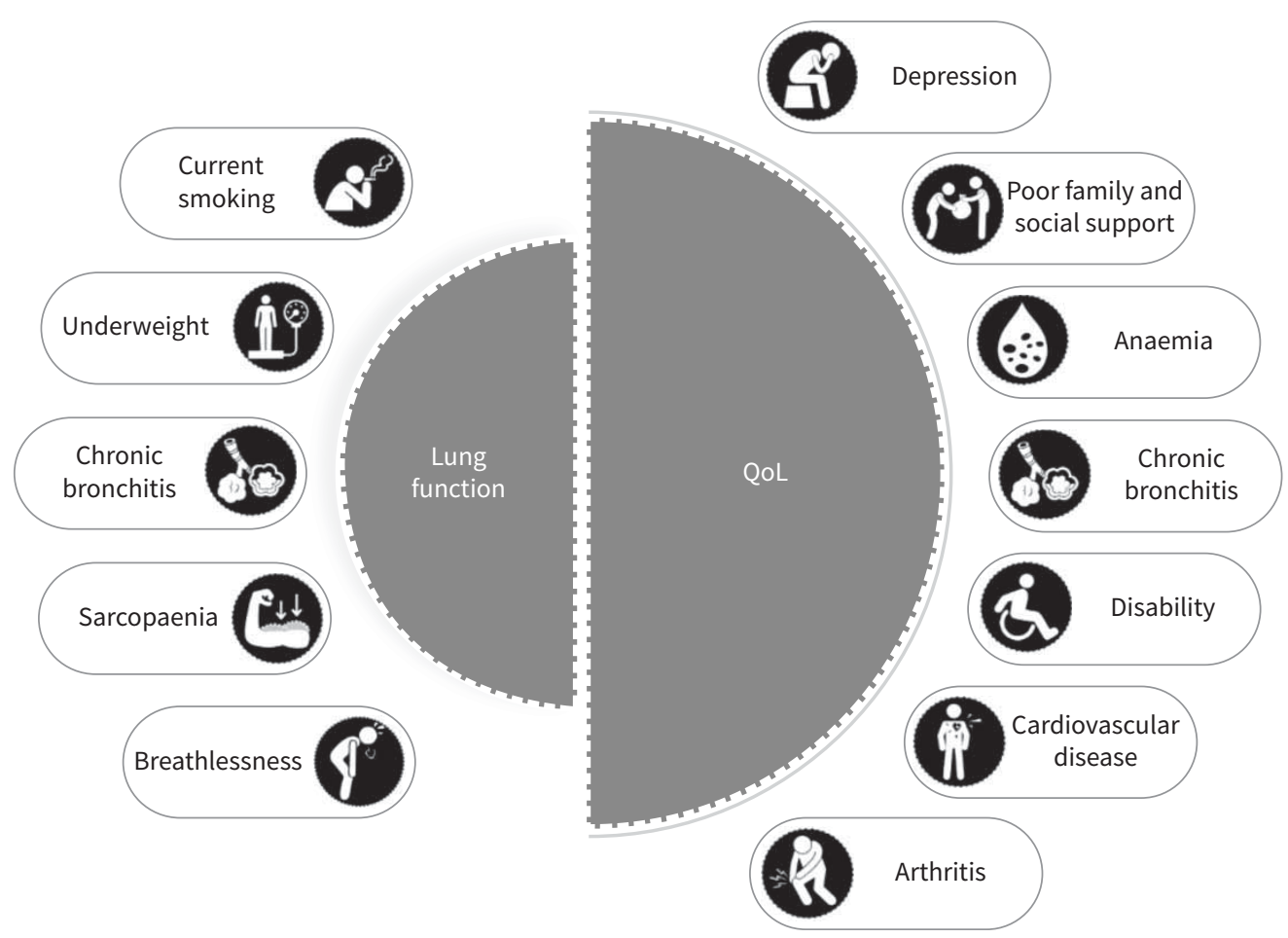

FIGURE 2 Treatable traits associated with decline in lung function and quality of life (QoL).

\section{Discussion}

This longitudinal study assessed the prevalence of numerous TTs and identified the traits that independently predicted decline in lung function and QoL among older people with or without COPD. All TTs had a higher prevalence among individuals with COPD, demonstrating the additional burden experienced by people with COPD compared to their counterparts. Importantly, we identified core TTs in COPD and non-COPD groups that independently predicted decline in lung function and QoL.

The current analysis highlights the fact that the decline in different outcomes, whether QoL or lung function, is associated with different traits. From 21 identified traits, five (including chronic bronchitis, breathlessness, underweight, sarcopaenia and current smoking) were associated with decline in lung function. For QoL there were seven traits (including chronic bronchitis, CVD, arthritis, depression, anaemia, disability, and poor family and social support) that independently predicted decline (figure 2) amongst individuals with COPD.

These findings are particularly important when making clinical decisions and in the design of interventions for TTs. This work advances knowledge of a precision medicine approach in COPD management and provides novel insights into the design of future trials targeting TTs and their implementation in clinical practice. Future studies should also investigate the impact of these traits on exacerbations, diseas e modification, healthcare utilisation and survival.

Our analyses showed that the traits of chronic bronchitis, breathlessness, underweight, sarcopaenia, and current smoking were associated with a decline in lung function among people with COPD. Various pharmacological and non-pharmacological strategies can be used for managing these traits. For instance, roflumilast [16], mucolytics (e.g. N-acetylcysteine and erdosteine) [17] and macrolides [18] have proven to be effective in reducing exacerbations among patients with COPD who have chronic bronchitis. Similarly, breathing retraining may improve lung function and breathlessness in patients with COPD [19]. Healthy dietary patterns, increasing consumption of fruits and vegetables along with vitamin $\mathrm{D}$, vitamin $\mathrm{E}, \mathrm{n}-3$ polyunsaturated fatty acids (PUFAs), essential amino acids, L-carnitine and whey protein, particularly when used in combination with a pulmonary rehabilitation program, improves/maintains lung function among individuals with COPD and should be a treatment priority in underweight individuals [20].

Furthermore, respiratory muscle training or resistance training may improve lung function and dyspnoea in patients with COPD who have sarcopaenia [21,22]. Smoking cessation plays a vital role in decreasing the decline in lung function associated with smoking [23]. It not only leads to a decreased risk of 
developing CVD and several types of cancer, but also increases the life expectancy of an individual [24]. A comprehensive and multidisciplinary approach is necessary for identifying the needs of people with COPD and this may have a positive effect on lung function and other health outcomes.

COPD can cause severe impairment of QoL resulting in a long-term, distressing burden. Thus, improving general QoL is crucial from a patient's perspective [25]; unfortunately, this goal has largely not been achieved despite advances in pharmacotherapy [26]. There is little agreement regarding the factors responsible for excessive decline in QoL. Our analyses showed that seven traits were associated with excessive decline in general QoL and among the strongest predictors were depression and poor family and social support. However, in the absence of a multidimensional assessment, depression remains the most common yet least recognised undertreated comorbidity in COPD, with a prevalence of 17-44\% [27]. This negatively affects the evolution of the respiratory disease and the patient's general QoL. Our results confirm the negative association between depression and QoL, and emphasise the need to recognise depression as a core trait in COPD. Appropriate strategies must be developed and implemented for diagnosing and managing depression, which may in turn improve QoL among people with and without COPD.

Poor family and social support in people with and without COPD predicted excessive decline in QoL. Strong social networks, along with social support, have a pivotal role in maintaining good health and QoL [28]. Studies have shown that social support improves health, motivates an individual to adhere to treatment and reduces depression, anxiety, hospitalisation and mortality, as well as increasing life span, functional capacity and willingness to take part in self-care activities [29, 30]. Strategies such as support groups, family therapy and multidisciplinary teams, including social workers, may be beneficial in patients with and without COPD.

Many of the predictors of lung function and QoL changes were significant and pointed in the same direction in both COPD and non-COPD groups. However, the associations were stronger in the COPD group. These findings indicate the importance of multidimensional assessment, not only among people with COPD but also among older patients with other chronic diseases.

This study has several strengths. To the best of our knowledge, it is the first comprehensive longitudinal study of TTs and their associations with health status among individuals with COPD, encompassing 21 TTs. The data were obtained from a large population-based prospective cohort, allowing minimum selection bias and thus increasing the generalisability of the findings. There were also some limitations, however. Data pertaining to exacerbations were not captured in the ELSA cohort and thus we were unable to evaluate exacerbations as a pertinent outcome of COPD along with lung function and QoL. Lung function data were based on pre-bronchodilator spirometry and thus some data with reversible airway obstruction or mixed airway disorder were possibly included in the COPD cohort. The number of traits assessed was restricted by the information available from the ELSA data set and thus not all traits in COPD reported in the literature could be assessed. We did not have medication data and could not adjust for the effects of treatment on outcomes. QoL was assessed using a generic scale, not one specific to COPD; however, this allowed exploration of the associations of traits in a non-COPD sample. Finally, the validity of physician or participant self-reports was not established. Nevertheless, the repeated, structured questionnaires were designed to reduce inaccuracy and to deal with uncertainty in participant recall.

\section{Conclusions}

Our study provided information regarding TTs contributing to decline in lung function and general QoL among individuals with COPD. Targeting the pulmonary traits of chronic bronchitis and breathlessness, the extra-pulmonary traits of underweight, sarcopaenia and depression, and the behavioural/life-style traits of smoking and poor family and social support may significantly improve health outcomes in people with COPD. Future studies should investigate holistic interventions targeting those core traits using a precision medicine approach and their short-term and long-term effects on health outcomes in COPD.

Acknowledgements: M.R. Sarwar acknowledges the support of an Australian Government Research Training Program scholarship. We wish to thank the English Longitudinal Study of Ageing investigators and study participants.

Ethics approval: The ELSA has been approved by the National Research Ethics Service (London Multicentre Research Ethics Committee: MREC/01/2/91). All participants gave written informed consent. Approval has also been obtained from the ELSA investigators and the Monash University Human Research Ethics Committee (MUHREC-22030) for the secondary analyses of the data.

Data availability: The raw data on which the conclusions of this manuscript rely are available upon reasonable request. The overall data results are available as part of the manuscript and its supplementary tables. However, if individual data points are needed these can be provided in response to a reasonable request. 
Author contributions: J. George, M.J. Abramson, V.M. McDonald and M.R. Sarwar conceived the research idea. Statistical analyses were conducted by M.R. Sarwar and E. Paul. The findings were interpreted and the first draft written by M.R. Sarwar. All authors contributed to the drafting and revision of the manuscript, and approved the final version for publication.

Conflict of interest: M.R. Sarwar has nothing to disclose. V.M. McDonald reports grants, personal fees and nonfinancial support from GSK and AstraZeneca, grants from NHMRC, Cyclopharm and Ramaciotti, and nonfinancial support from Menarini, outside the submitted work. M.J. Abramson reports grants and personal fees from GSK, grants from Pfizer and Boehringer Ingelheim, and nonfinancial support from Sanofi, outside the submitted work. E. Paul has nothing to disclose. J. George reports grants from GSK, Pfizer and Boehringer Ingelheim, outside the submitted work.

Support statement: No funding has been received for the preparation of this manuscript or has influenced the decision to submit it for publication. Funding for the ELSA was provided by the National Institute of Aging and a consortium of UK Government departments coordinated by the Office for National Statistics. Funding information for this article has been deposited with the Crossref Funder Registry.

\section{References}

1 McDonald VM, Osadnik CR, Gibson PG. Treatable traits in acute exacerbations of chronic airway diseases. Chron Respir Dis 2019; 16: 1479973119867954.

2 Agustí A, Bafadhel M, Beasley R, et al. Precision medicine in airway diseases: moving to clinical practice. Eur Respir J 2017; 50: 1701655.

3 Agusti A, Bel E, Thomas M, et al. Treatable traits: toward precision medicine of chronic airway diseases. Eur Respir J 2016; 47: 410-419.

4 McDonald VM, Fingleton J, Agusti A, et al. Treatable traits: a new paradigm for 21st century management of chronic airway diseases: Treatable Traits Down Under International Workshop report. Eur Respir J 2019; 53: 1802058.

5 Maurer J, Rebbapragada V, Borson S, et al. Anxiety and depression in COPD: current understanding, unanswered questions, and research needs. Chest 2008; 134: 4 Suppl., 43S-56S.

6 Hiles SA, Gibson PG, Agusti A, et al. Treatable traits that predict health status and treatment response in airway disease. J Allergy Clin Immunol Pract 2020; 9: 1255-1264.

7 Downs SH, Brandli O, Zellweger JP, et al. Accelerated decline in lung function in smoking women with airway obstruction: SAPALDIA 2 cohort study. Respir Res 2005; 6: 45.

8 Rennard SI, Locantore N, Delafont B, et al. Identification of five chronic obstructive pulmonary disease subgroups with different prognoses in the ECLIPSE cohort using cluster analysis. Ann Am Thorac Soc 2015; 12: 303-312.

9 Steptoe A, Breeze E, Banks J, et al. Cohort profile: the English longitudinal study of ageing. Int J Epidemiol 2013; 42: $1640-1648$.

10 Miller MR, Hankinson J, Brusasco V, et al. Standardisation of spirometry. Eur Respir J 2005; 26: 319-338.

11 Quanjer PH, Stanojevic S, Cole TJ, et al. Multi-ethnic reference values for spirometry for the 3-95-yr age range: the global lung function 2012 equations. Eur Respir J 2012; 40: 1324-1343.

12 Padberg I, Schneider A, Rohmann JL, et al. Impact of COPD and anemia on motor and cognitive performance in the general older population: results from the English longitudinal study of ageing. Respir Res 2020; 21: 40.

13 Singh D, Agusti A, Anzueto A, et al. Global strategy for the diagnosis, management, and prevention of chronic obstructive lung disease: the GOLD science committee report 2019. Eur Respir J 2019; 53: 1900164.

14 Hyde M, Wiggins RD, Higgs $\mathrm{P}$, et al. A measure of quality of life in early old age: the theory, development and properties of a needs satisfaction model (CASP-19). Aging Ment Health 2003; 7: 186-194.

15 McDonald VM, Clark VL, Cordova-Rivera L, et al. Targeting treatable traits in severe asthma: a randomised controlled trial. Eur Respir J 2020; 55: 1901509.

16 Martinez FJ, Calverley PM, Goehring UM, et al. Effect of roflumilast on exacerbations in patients with severe chronic obstructive pulmonary disease uncontrolled by combination therapy (REACT): a multicentre randomised controlled trial. Lancet 2015; 385: 857-866.

17 Poole P, Sathananthan K, Fortescue R. Mucolytic agents versus placebo for chronic bronchitis or chronic obstructive pulmonary disease. Cochrane Database Syst Rev 2019; 5: CD001287.

18 Herath SC, Normansell R, Maisey S, et al. Prophylactic antibiotic therapy for chronic obstructive pulmonary disease (COPD). Cochrane Database Syst Rev 2018; 10: CD009764.

19 Gimenez M, Servera E, Abril E, et al. Comprehensive directed breathing retraining improves exertional dyspnea for men with spirometry within normal limits. Am J Phys Med Rehabil 2010; 89: 90-98.

20 Yang IA, George J, Jenkins S, et al. The COPD-X plan: Australian and New Zealand guidelines for the management of chronic obstructive pulmonary disease 2021. Version 2.63 (February 2021). https://copdx.org.au/ copd-x-plan/

21 Neves LF, Reis MH, Plentz RD, et al. Expiratory and expiratory plus inspiratory muscle training improves respiratory muscle strength in subjects with COPD: systematic review. Respir Care 2014; 59: 1381-1388.

22 Liao WH, Chen JW, Chen X, et al. Impact of resistance training in subjects with COPD: a systematic review and meta-analysis. Respir Care 2015; 60: 1130-1145.

23 Anthonisen NR, Connett JE, Kiley JP, et al. Effects of smoking intervention and the use of an inhaled anticholinergic bronchodilator on the rate of decline of $\mathrm{FEV}_{1}$ : the Lung Health Study. JAMA 1994; 272: $1497-1505$.

24 Taylor DH Jr, Hasselblad V, et al. Benefits of smoking cessation for longevity. Am J Public Health 2002; 92: 990-996.

25 King CR, Hinds PS, eds. Quality of life: from nursing and patient perspectives. 3rd Edn. Sudbury, Jones \& Bartlett Learning, 2012.

26 Zamzam MA, Azab NY, El Wahsh RA, et al. Quality of life in COPD patients. Egypt J Chest Dis Tuberc 2012; 61 281-289. 
27 Albrecht JS, Park Y, Hur P, et al. Adherence to maintenance medications among older adults with chronic obstructive pulmonary disease. The role of depression. Ann Am Thorac Soc 2016; 13: 1497-1504.

28 Bélanger E, Ahmed T, Vafaei A, et al. Sources of social support associated with health and quality of life: a cross-sectional study among Canadian and Latin American older adults. BMJ open 2016; 6: e011503.

29 Kara Kaşıç̧ı M, Alberto J. Family support, perceived self-efficacy and self-care behaviour of Turkish patients with chronic obstructive pulmonary disease. J Clin Nurs 2007; 16: 1468-1478.

30 Chen Z, Fan VS, Belza B, et al. Association between social support and self-care behaviors in adults with chronic obstructive pulmonary disease. Ann Am Thorac Soc 2017; 14: 1419-1427. 